
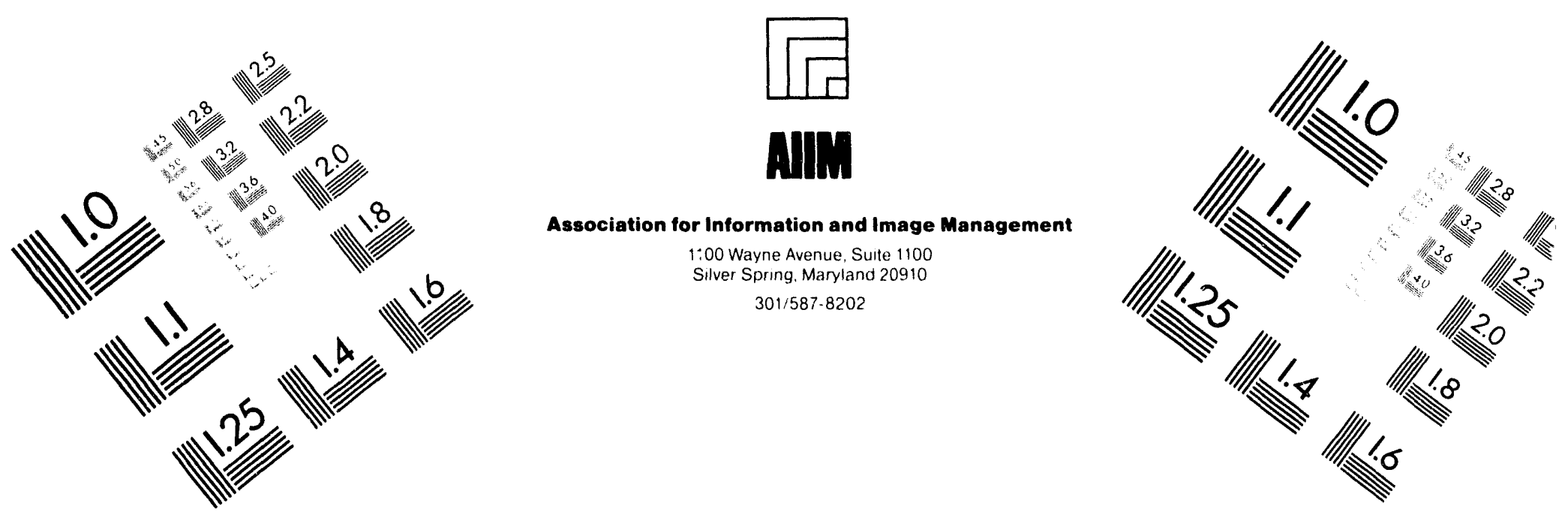

\title{
Centimeter
}

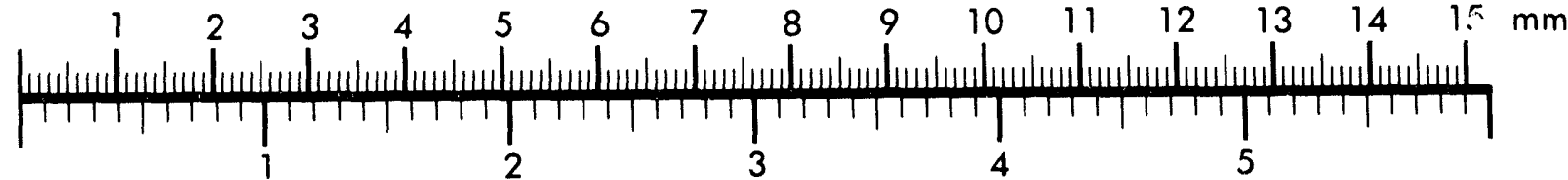

Inches
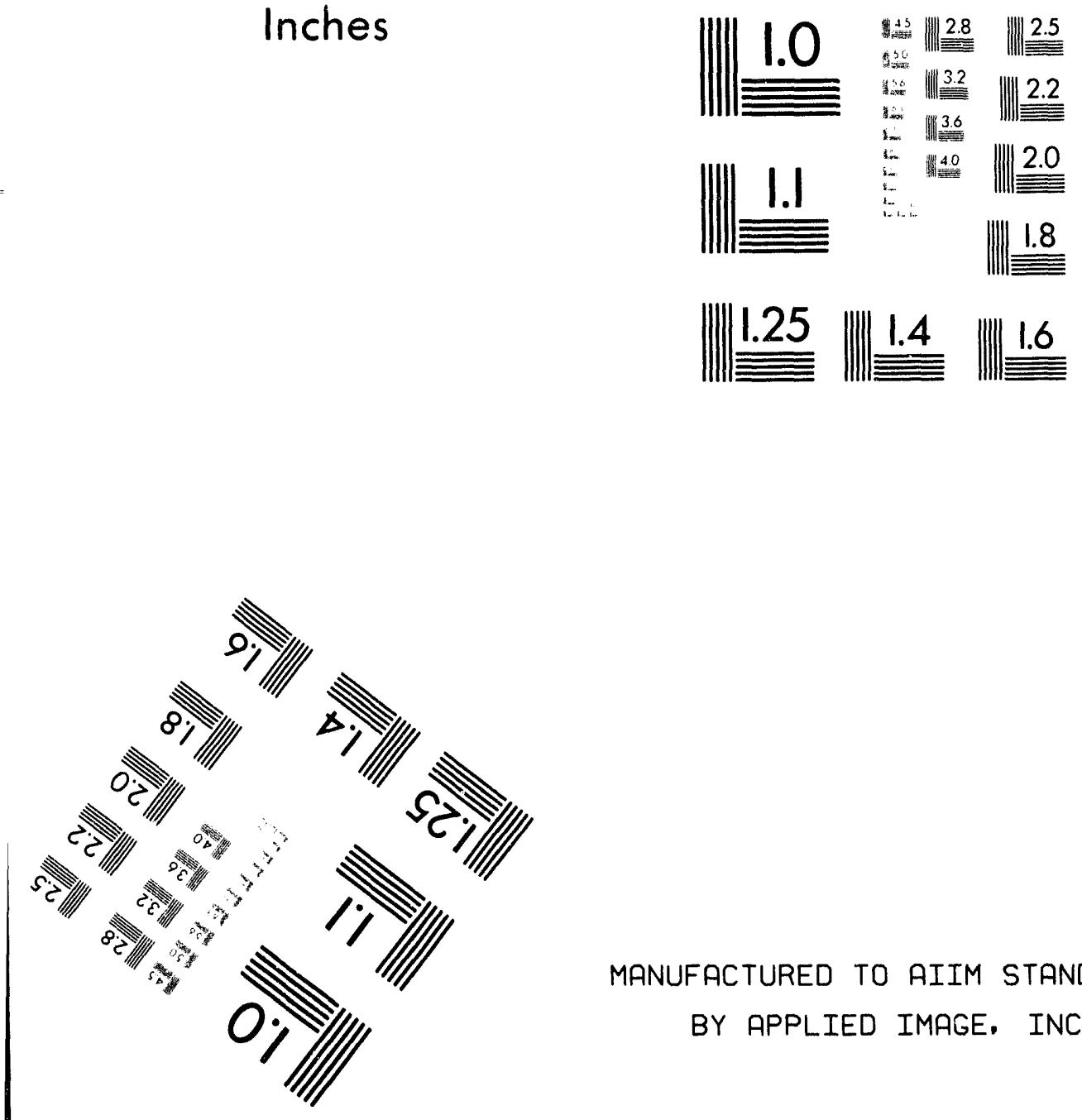

MANUFACTURED TO AIIM STANDARDS

BY APPLIED IMAGE. INC.

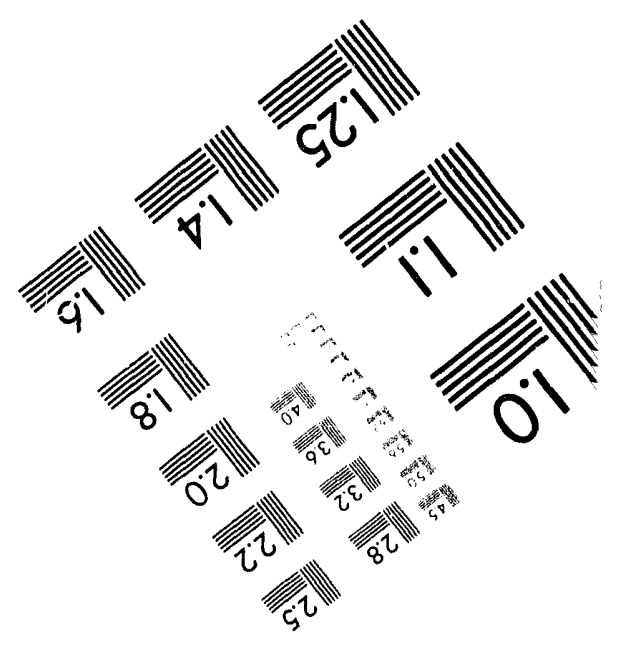



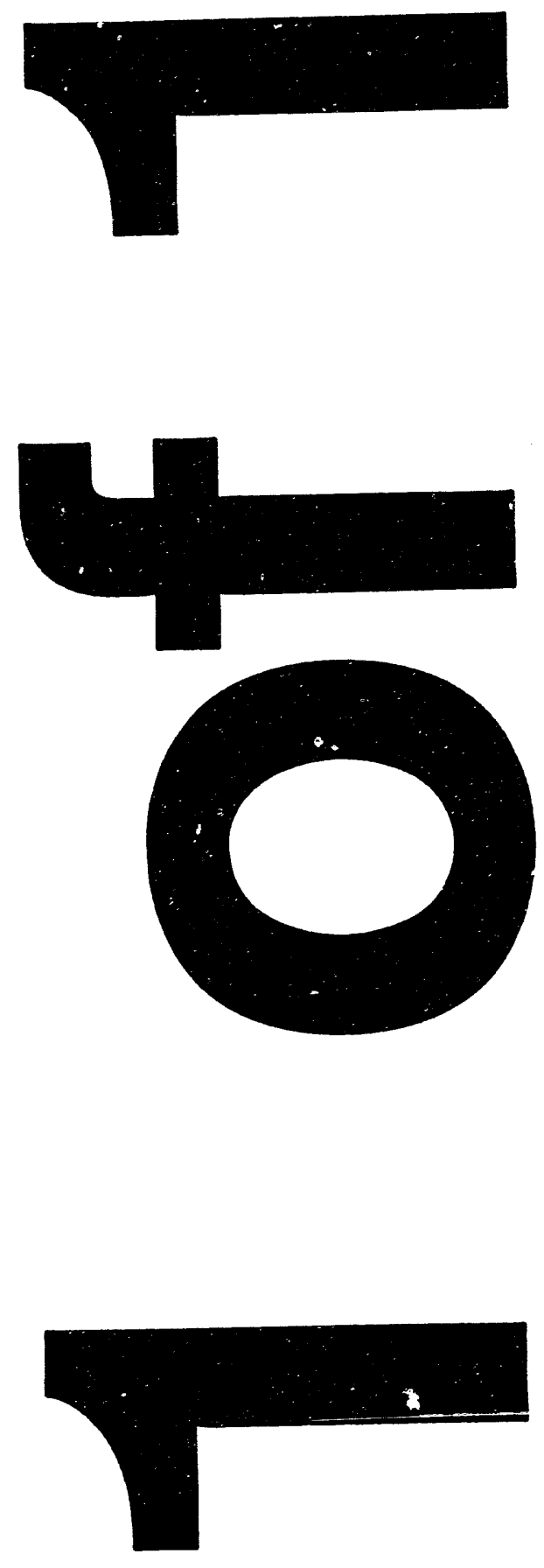

" I 


$$
\text { DOE/ER/25046-..TI }
$$

\author{
Measuring Performance of \\ Parallel Computers \\ Interagency Agreement No. DE-AI05-87ER25046 \\ Final Report \\ Francis Sullivan \\ Center for Computing and Applied Mathematics \\ National Institutes of Standards and Technology \\ Gaithersburg, Maryland 20899
}

\title{
Preface
}

\section{Performance Measurement.}

We have developed a taxonomy of parallel algorithms based on data motion and example applications have been coded for each class of the taxonomy. Computational benchmark kernels have been extracted for several applications, and detailed measurements have been performed.

\section{Algorithms for massively parallel SIMD machines.}

Measurement results and computational experiences indicate that top performance will be achieved by "iteration" type algorithms running on massively parallel SIMD machines. Reformulation as iteration may entail unorthodox approaches based on probabilistic methods. We have developed such methods for some applications.

Research has been carried out by staff from the Center for Computing and Applied Mathematics (CCAM) and the Institute for Computer Sciences and Technology (ICST). Because of its experience in scientific computing, CCAM emphasized the applications software side of the work. ICST has been responsible for the hardware measurements program. Close contact has been maintained with other programs in parallel computing. In particular, faculty from the University of Maryland and the University of Colorado have been involved.

In the following sections we discuss our approach to performance measurement, describe the taxonomy and measurements which have been made, and report on some general conclusions which can be drawn from the results of the measurements. The appendix contains a summary of activities and publications associated with the proiect.

\section{DISCLAIMER}




\section{Discussion of Approach}

The major questions needing to be answered are

- How can codes be improved?

- What kind of codes should be run on what kind of machine?

Our point of view is that data motion is the principal determining factor in the performance of algorithms on parallel machines.

Our approach to performance measurement has been to first develop a taxonomy for algorithmic paradigms based on how the data moves during the calculation, rather than on what the specific computational steps are. Thus, from this viewpoint, "Ising spin simulations" occur in two different places in the taxonomy as "applications," depending on which algorithm is to be used. Benchmark codes typical of applications in each class of the taxonomy have been developed and implemented on different parallel computers in order to explore the relations between the taxonomy and the "machine event level" of a layered framework or spectrum.

The upper level is the applications which dictate the required computational load. The lowest level is machine events, memory and communication links, having capabilities that should satisfy the upper level requirements. Brief descriptions of the layers follow:

1. Applications. This layer provides broad descriptions of problem areas, such as fluid dynamics, statistical mechanics, and quantum chemistry. While each application area does have associated "typical computations," the real issues are algorithmic features which cut across several problem types.

2. Algorithmic Paradigms. This is the level beneath the applications. There is a taxonomy of algorithmic paradigms, each common to many level 1 applications. These include iteration on regular grids (emphasizing local data dependencies), searching ordered structures (emphasizing global data dependencies and implied global constraints), event scheduling (of independent processes), traversing graphs (recursive local dependencies and global constraints), and random walks (problem-determined dynamic global constraints).

3. Process Mechanisms and Organization. The next lower level of detail concerns the use of process mechanisms to provide an abstract model of 
processes divorced from the details both of the original problem, and algorithmic and coding features not related to processes. These include process creation costs, the pattern and volume of data communication, and general parallel programming service overheads. Some of the process communication pattern may be inherited from an algorithmic paradigm, while other parts evolve from constraints of the machine architecture. Synchronization methods and modes of information transmission are the two principal concerns.

4. Code and Machine Event Level. Here the general balance of the parallel architecture is tested with the execution of a program. The detail of measurement on this level is fine-grained, in the microsecond range (or less), with items of interest typically being bus waits, cache hit ratios, time-tolock, etc. These details cannot be obtained by software methods at higher levels. Nevertheless, such measurements are essential because observations at higher levels in the model need to be correlated with what is actually occurring at the machine level.

We have chosen the target machines as representatives of the three main classes of parallel architectures: loosely coupled, message passing machines; tightly coupled, shared memory machines; and SIMD machines. The computers have been (or will be) instrumented to allow detailed measurement and analysis of machine events, such as resource utilization and data motion. The measurements lead to identification of the "kernel" process mechanisms in each benchmark, and also result in benchmark implementation principles to optimize performance.

Our first target machine was a tightly coupled, shared memory machine, the Sequent B-8000, originally with six processors and now with twelve. NBS (now NIST) has designed and implemented two measurement systems for this computer. The first version of the measurement system allowed collection of time-stamped execution traces in which use of processors and processes is identified [12]. The current version of the NIST measurement system is now available on the Sequent. This allows collection of more detailed resource utilization information, such as cache hit ratio and bus access latency, vs. progress in the execution of the test programs.

Our second target machine is a loosely coupled, message passing machine, an Intel iPSC with 32 processors. This has also been instrumented with specialized hardware developed at NIST. Very low overhead has been achieved by attaching the hardware to one of the computer busses. The user takes measurements by 
making a simple subroutine call, which places one word of data on the bus. The specialized hardware takes the word off the bus, attaches the time and the proces-

sor number, and logs the data. The only overhead to the system is one subroutine call and one word of extra bus traffic.

In the fall of 1988, we will purchase and begin to instrument an SIMD machine, a DAP 510 with at least 1024 processors.

The current NIST measurement systems produce tabular data which can be displayed in charts or graphs and which provide indication of the use of system resources as a function of progress in the execution of code. The results of work on parallel programming techniques guide us in the selection of parameter combinations to display. These displays of program sections and constructs and the sources of their inefficiency permit targets for improvement to be identified.

In summary, this study results in improved understanding of the relations among data flow, machine configuration, and algorithm efficiency and it provides information on the choice of machine architecture characteristics to be measured. In particular, it tests the utility of the spectrum and the taxonomy. For example, a class of the taxonomy that measurements predict to be well suited to loosely coupled architectures should perform well on several different bosely coupled machines.

\section{Explaination of Taxonomy and Examples of Measure- ments}

In this section we discuss our taxonomy based on data motion and sketch some examples of applications and measurements performed on computational kernels.

A. Iteration on Regular Grids. The paradigm is

- For all grid points, $i$, find a new $v_{i}$ from old nearest neighbors $v_{i+1}, v_{i-1}$, $v_{i+l}, v_{i-l}$.

The most obvious algorithms in this category are those associated with finite difference approximations to partial differential equations. However, there are other examples. In particular, the FFT algorithm can be written so that successive steps consist of a sequence of nearest neighbor updates, where the old values are multiplied by the appropriate complex roots of unity. Another example is a sorting algorithm, DiamondSort [5], which performs well on vector machines. Because of the constraint of vector architecture, the method is designed to be 
"non-contingent" - the flow of control does not depend on the particular sequence to be sorted, only on its length. The kernel process is a sequence of vector merges which take the form of nearest neighbor updates.

Ising spin simulations occur in many areas of statistical physics, such as studies of phase transitions, alloy solidification, and domain growth. Spin system simulations have attracted the attention of researchers since the earliest days of electronic computation [11]. They provide a very good example of how, from the point of view of algorithm design, two apparently disparate problem types can be attacked by almost the same techniques. For standard methods, the kernel process is "iteration on regular grids." This observation suggests parallel algorithms similar in spirit to those used for solving elliptic pde's by finite difference approximations [4].

\section{Ising Spin Exchange on a Shared Memory Machine}

Associating processors with spin sites in a one-one manner is not practical for a shared memory machine. Instead of mapping sites to processors, we use the fact that the expressions for the energy associated with a site are similar in form to the central difference approximation used to solve the Poisson equation, where a red-black ordering is often used. Since no two red sites are nearest neighbors, all red sites can be updated "simultaneously." These values can then be used to update the black sites, and so-forth, alternating on each iteration between red and black sites. The same idea can be applied to modify the method of [11]. At each step, one of the two colors is chosen at random and then spin flips are attempted at all sites of that color [14]. The ordering of which sites to do first is immaterial.

In spin-exchange simulations, at least five colors seem to be required, and sixteen have been used for large-scale calculations on the Cyber 205. The result is an extremely fast vector code [2]. The five-coloring is as follows:

$\begin{array}{llllllllll}1 & 2 & 3 & 4 & 5 & 1 & 2 & . & . & . \\ 3 & 4 & 5 & 1 & 2 & 3 & 4 & . & . & . \\ 5 & 1 & 2 & 3 & 4 & 5 & 1 & . & . & . \\ 2 & 3 & 4 & 5 & 1 & 2 & 3 & . & . & . \\ 4 & 5 & 1 & 2 & 3 & 4 & 5 & . & . & . \\ 1 & 2 & 3 & 4 & 5 & 1 & 2 & . & . & . \\ . & . & . & . & . & . & . & . & . & . \\ .\end{array}$

Two methods have been implemented on the Sequent, using five processors. 
Version 1: "Synchronized"

Randomly choose one of the five colors, and randomly choose a direction from among \{ up, down, left, right \}. All pairs of the chosen type can be worked on simultaneously by all the processors, dividing up the sites among the processors in any desirable way. Evaluating all pairs of a given type is called a "sweep" of that color. Sites of a given color can be swept in sequential order without introducing false dynamics into the simulation. The processors must be synchronized at the start of a color, when the pair type is chosen, to avoid memory conflicts; then no "locking" needs to be done. Tests indicate that for a five processor machine one can expect a speedup factor of 3.5 to 4.5 over the execution time for a single processor.

Version 2: "Independent"

If processes are not synchronized, each processor can work independently on a pair type. A memory "lock" must be used whenever spins are exchanged, because the the physics dictates that the balance between " 1 " and " -1 " spins must be maintained. It is possible that, because of memory conflicts, "old" spin information from one color will be used in some evaluations for another color. Numerical experiments and measurements seem to indicate that independent asynchronous evaluation causes no difficulties and is more efficient than synchronizing on the particular shared memory computer tested.

Instead of locking on every exchange, it is possible for each processor to save a list of needed exchanges and wait until the end of each sweep to exchange. Only one lock per sweep per processor is needed, but the overhead is higher.

For the independent case, a proof that the correct distribution is sampled will depend on the computer architecture, because the resolution of memory conflicts determines the age of the data used. The method of chaotic relaxation uses similar algorithms which have been shown to be correct [6].

\section{B. Searching Ordered Structures.}

- Find all $x$ such that;

$$
m_{i} \leq x_{i} \leq M_{i} ; \text { for all } i=1, \ldots, k
$$

It has been known for a long time that sorting and searching procedures are pervasive in applications of computers to "non-scientific" areas. In fact, from the point of view of amount of computer time used, they are central to many scientific applications. In molecular dynamics codes, for example, searching can be used 
as the basis of a method for constructing "neighbor tables" that greatly increase the efficiency of the code [8]. Sorting can also be used to give a fast algorithm for computation of fractal dimensions [7].

C. Event Scheduling.

- Assign initial ranks.

- Do the rank-1 task.

- Re-order the ranks based on outcome.

Typical applications of algorithms in this class include adaptive quadrature, and one version of algorithms for Ising spin simulations. In the case of adaptive quadrature, the region of integration is subdivided into pieces, and an estimate of the relative error is made for each piece. The piece giving the largest error is the rank-1 task. To do the task we subdivide that piece and do a more accurate quadrature on the new subdivisions. This generates new tasks and ranks, etc. A similar idea can be used to speed up Ising spin simulations. Spin sites can be ranked according to the change in total energy which would result from reversing the spin at that site. The ranks are modified by the appropriate probabilities, but the principle is the same; a rank-1 task is performed (i.e. a spin is reversed) and the new ranks are assigned.

In both cases, sets (quadrature regions, spin sites) are continually being formed and ranked, and the item of interest is the current rank-1 set. In actual codes the set manipulations often take more time than the "calculations" (computing error estimates, evaluating energy changes). Data structures and algorithms which can be used for the set manipulations include heaps and more general order trees. The "kernel" process is "access the tree." Adapting such a kernel to a parallel machine raises interesting questions. On a loosely coupled architecture, for example, one probably should not try assigning sets to individual processors, because the membership of sets is constantly changing and so a lot of communication between processors would be required. On a tightly coupled machine, the usual worries about memory conflicts arise. In either case dealing with the set accessing problem is the central issue.

We have performed measurements on an event scheduling version of the quicksort algorithm. The idea is to keep to endpoints determined by subdividing lists on a heap which is accesses by all processes. A typical process accesses the heap and then either subdivides the indicated list or sorts it at once if the length is below a predetermined threshold. This is expressed in the following: 
DO WHILE forever

lock-heap

IF heap not $\phi$ THEN

busy (id)=.true.

$(l, r) \Leftarrow h($ top $) ;$ delete $h($ top $)$

unlock-heap

BEGIN partition $(l, r)$

IF $r-l<n$ THEN

$$
\operatorname{sort}(l, r)
$$

ELSE

compute $l<m<r$; place $x(m)$

lock-heap

$h \Leftarrow(l, m),(m+1, r)$

unlock-heap

ENDIF

END partition

busy $($ id $)=$.false.

ELSE

unlock-heap

\section{ENDIF}

\section{END DO WHILE}

Note that the "busy" vector is needed as a stopping condition, because the heap may be temporally empty even though the sort is not complete.

In the first set of measurements, a single process was used so that an execution profile could be obtained. The heap was ordered by "shortest list first."

Version 1: Shortest list at top of heap
\begin{tabular}{|l|r|r|}
\hline Interval & Samples & Mean Time \\
\hline Sorting & 2 & $7 \mathrm{M}$ \\
Access heap & 6600 & 212 \\
Divide list & 3301 & $3 \mathrm{~K}$ \\
Sort list & 3303 & 764 \\
Create entry & 1403 & 18 \\
\hline
\end{tabular}

The same single process measurements were done for the "longest list first" order. This keeps the processors busy, but increases the overhead associated with locking the heap. 
Version 2: Longest list at top of heap

\begin{tabular}{|l|r|r|}
\hline Interval & Samples & Mean Time \\
\hline Sorting & 2 & $8.5 \mathrm{M}$ \\
Access heap & 6600 & 503 \\
Divide list & 3301 & $3 \mathrm{~K}$ \\
Sort list & 3303 & 968 \\
Create entry & 1403 & 16 \\
\hline
\end{tabular}

In fact, it is interesting to note that as processes are added, the time-to-lock dominates the execution time, i.e. as the number of processes increases, data communication determines execution time.

\begin{tabular}{|r|r|r|r|}
\hline Proc. & Total & Heap & Lock \\
\hline 1 & $6.1 \mathrm{M}$ & 691 & 36 \\
2 & $3.9 \mathrm{M}$ & 715 & 306 \\
3 & $3.5 \mathrm{M}$ & 728 & 918 \\
4 & $3.5 \mathrm{M}$ & 735 & 1728 \\
5 & $3.5 \mathrm{M}$ & 724 & 2435 \\
6 & $3.6 \mathrm{M}$ & 755 & 3380 \\
\hline
\end{tabular}

\section{Traversing Graphs}

Assume that $v$ is a node of a graph and that $L(v)$ is a list of nodes that can be reached from $v$ in a single step. The traversal paradigm is:

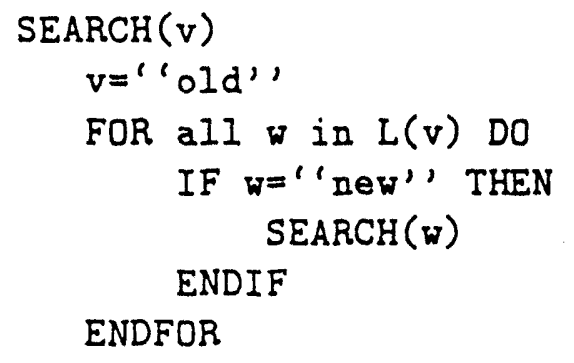

The pseudo-code is a recursive expression of the idea of "visiting" every node of a graph without repetitions. Algorithms in this category include techniques such as post-order traversal of binary trees and, more generally, depth-first search of directed and undirected graphs. It finds application in many areas ranging from parsing routines for language processors to determination of connected components of graphs. A "chaotic" modification of the basic algorithm well suited to 
shared memory machines consists of spawning separate processes to search all $w$ in $L(v)$.

An interesting application of traversal which has been coded for measurement comes from a method for triangulation in 3-D based on ordertype information. The point $X_{l}$ is on the positive side of the plane determined by $X_{i}, X_{j}, X_{k}$ in case the determinant

$$
\left|\begin{array}{cccc}
1 & x_{i, 1} & x_{i, 2} & x_{i, 3} \\
1 & x_{j, 1} & x_{j, 2} & x_{j, 3} \\
1 & x_{k, 1} & x_{k, 2} & x_{k, 3} \\
1 & x_{l, 1} & x_{l, 2} & x_{l, 3}
\end{array}\right|
$$

is positive. The ordertype determines the function $\alpha(X, Y, Z)$ defined to be the first $W$ encountered when the $(X, Y, Z)$ plane is rotated in the positive direction. This is central to triangulation in $3-D$, because the tetrahedron $(X, Y, Z, W)$ has no points in its interior an so can be used in a triangulation. If $\left(V_{1}, V_{2}, V_{e}\right)$ is a boundary triangle then, by fixing $V_{e}$, tetrahedra of one "cap" (= those tetrahedra having $V_{e}$ as a vertex) of the triangulation can be determined by a traversal of the $\alpha\left(., ., V_{e}\right)$ graph. This is done by the recursive HANDLE procedure below.

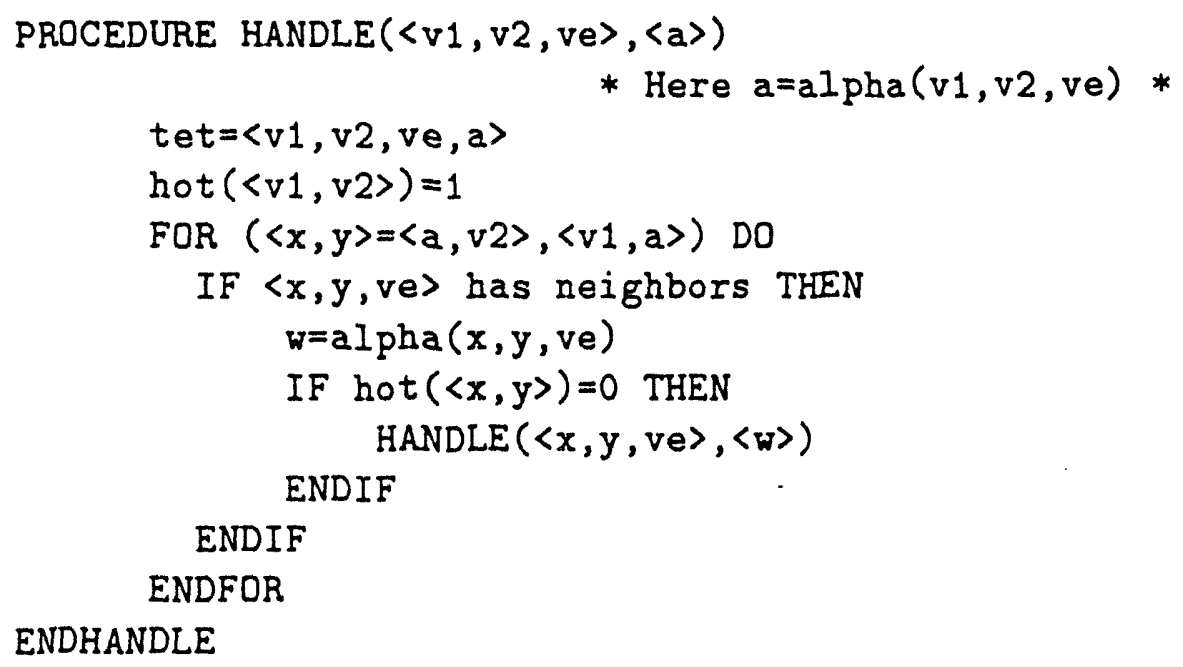

Here 'tet' is a tetrahedron and "hot" means that an edge has been seen before (="old" in the paradigm). A triangle has "neighbors" if it is not on the boundary. After HANDLE has been done, $V_{e}$ can be erased and the process re-started with a new boundary triangle. To estimate the total number of tetrahedra found by this 
method we can integrate to determine the total number of triangles in all caps giving,

$$
\text { Total }=\int S(r) d r=\int k^{1 / 2} d k=O\left(n^{3 / 2}\right) .
$$

To see this, notice that if a cap involves $k$ points, we get $O(k)$ triangles. Therefore, the "surface area" $S(r)$ for radius $r=O\left(k^{1 / 2}\right)$ is $O(k)$ i.e.

$$
S(r) \sim k, r \sim k^{1 / 2}
$$

and so,

$$
d r \sim k^{-1 / 2} d k
$$

The result of the triangulation is a list of tetrahedra

$$
\mathcal{T}=\left(T_{1}, T_{2}, \ldots, T_{n}\right)
$$

determining the triangulation. Another graph traversal can be used to obtain a re-ordering:

$$
\mathcal{S}=\left(S_{1}, S_{2}, \ldots, S_{n}\right)
$$

such that, for all $1<i<n$

$$
S_{i} \cap \bigcup_{j<i} S_{j}
$$

is topologically a 2-ball and for $i=n$ a 2-ball or a 2-sphere. This is called a shelling of the triangulation. This is useful in applications where proximity information is required, because the shelling order numbers the tetrahedra so that there are no gaps or jumps.

\section{E. Random Walks}

By this we mean generalizations of the fundamental "importance sampling" method that originated in Monte Carlo calculations. This is the sequence: Try a move. If the move decreases the "energy," do it. If it increases the energy, do it with a pre-determined probability. The paradigm is expressed in the following pseudocode:

Chose "random" site

Compute $\Delta E$

IF $\triangle E \geq 0$ THEN

IF $r \leq P(\Delta E)$ THEN

change site

ENDIF 


\section{ELSE \\ change site \\ ENDIF}

This idea has found application in a wide range of problems, including simulation of diffusion limited aggregation, growth of random surfaces, motion of polymer chains, and percolation. As has already been mentioned, "classical" Monte Carlo calculations can be done most efficiently by iteration, rather than by random walk. On the other hand, through its incarnation as simulated annealing, random walk has been used for purely combinatorial calculations, such as the traveling salesman's problem.

Data motion questions are central to implementations of the "choose at random" and "change site" steps. We have coded a random surface model in which the list of available sites is maintained as an octree and a heap with pointers to the octree is used for choose at random. Other possibilities include AVL trees and splay trees.

\section{General Results and Observations}

Specific results have been obtained as a result of the development and detailed measurement of computational kernels for several classes of the taxonomy. In addition, as a consequence of carrying out the measurements, new approaches and new algorithms have been discovered for problems ranging from triangulation in 3-D to simulation of razdom surface growth. Recently, a deeper understanding of the taxonomy itself has begun to emerge, and some general principles of parallel algorithm design can now be proposed. Here we summarize a few observations concerning the taxonomy and begin to formulate some general principles of parallel algorithm design.

Stated briefly, the accumulated evidence supports the view that data motion determines performance on parallel machines. This motivates the search for general algorithms in which data motion is minimized. Algorithms from the class "iteration on regular grids" seem to be most amenable to minimization of data motion. The best performance for such algorithms will be obtained on massively parallel SIMD machines.

Our focus on data motion as the determinant of performance means that algorithms are first examined from the point of view of data dependencies. We 
have found that interprocess communication is always expensive to do on MIMD machines. In the case of tightly-coupled, shared memory machines, the communication requirement entails process synchronization and/or memory locking. For shared memory machines, it is best to avoid global synchronization by using "chaotic" methods when possible. This is illustrated by the measurements performed for iteration on the Sequent implementation of the Ising model. The overhead associated with synchronization of processes at the end of each iteration is much higher than that associated with locking memor" locations. The measurements on the event scheduling quicksort routine provide an example of how even the wait times associated with locking can cancel the advantages expected from multi-processor algorithms. For loosely coupled message passing machines, the rule-of-thumb seems to be "avoid passing .nessages!" Often this can be accomplished by adapting ideas developed for shared memory machines, because the data independence can be carried over.

In fact, very general methods for ensuring data independence are occasionally discovered, resulting in parallel algorithms which can be used for several classes of machines. The most dramatic illustrations of this are cases in which the core computation is "iteration on regular grids" or can be expressed as iteration. Thus, the use of colors leads to fast Ising codes on the Sequent Balance 8000, the Cyber 205 , and the Connection Machine. Naturally, important implementation details vary from machine to machine, but the underlying idea is the same in all cases. Coloring of grid sites decomposes the problem into relatively independent chunks. A striking example of reformulation as iteration is provided by the Diamondsort algorithm [5]. The task of sorting an array is reformulated as an iteration method having a data motion pattern similar to that used in the cyclic reduction method for solving linear systems. The result is an algorithm for which fast implementations have been developed on a variety of machines (e.g. Cyber 205, MPP, Connection Machine).

By now, it appears to be clear that formulation as iteration on regular grids offers the greatest opportunity for development of fast parallel algorithms. Searching ordered structures is a close second. Naturally, for significant problem classes, such formulations may be impossible. There almost certainly are situations in which the underlying problem has inherent global dynamic constraints and so is of class "graph traversal" or "random walk." However, in a surprising number of cases, a reformulation is possible. As has already been mentioned, even sorting can be turned into iteration. In addition, fractal dimension computations [7] and molecular dynamics [8] simulations are amenable to solution by searching 
methods.

Obtaining very large speedups from parallel architectures will require massive parallelism. The results of our work indicate that the move to massive parallelism has several implications for algorithm design. Because of the cost associated with communication and synchronization overheads, most massively parallel machines will have SIMD architectures. These have the advantage of being almost ideal for "iteration on regular grids" algorithms, and the disadvantage of nearly requiring an iteration type formulation for all problems. Such reformulations may take the form of "inexact looking but nevertheless correct (ILNC)" methods. For example, the use of colors in Ising simulations is not a strict implementation of the Metropolis algorithm and the chaotic method is even more non-standard. Nevertheless, both give accurate results. The data compression map used in fast computation of fractal dimensions gives accurate results, even though it does not preserve distances. A more sophisticated and less ad hoc formulation of ILNC methods will use ideas suggested by lattice gas theory.

\section{References}

[1] An Agenda for Improved Evaluation of Supercomputer Performance, National Research Council (1986).

[2] Amar, J. G., Sullivan, F. E., and Mountain, R. D., A Monte Carlo Study of Growth in the Two-Dimensional Spin-Exchange Kinetic Ising Model, Phys. Rev. B, 37 (1988).

[3] Bentley, J. L., Writing Efficient Programs, Prentice-Hall, Englewood Cliffs, N. J., 1982.

[4] Blue, J. L., and Sullivan, F. E., Ising Spin on a Shared Memory Machine: Computational Experience, Proc. Third SIAM Conf. on Parallel Processing for Scientific Computing, to appear.

[5] Brock, H., Brooks, B. and Sullivan, F. 1981. Diamond: A Sorting Method for Vector Machines., BIT, 21 (1982), pp. 142-152.

[6] Lubachevsky, B. D., and Mitra, D., A Chaotic Asynchronous Algorithm for Computing the Fixed Point of a Nonnegative Matrix of Unit Spectral Radius, J. ACM 33 (1986), pp. 130-150. 
[7] Hunt, F. and Sullivan, F., Efficient Algorithms for Computing Fractal Dimensions, Los Alamos National Laboratory (1986).

[8] Mountain, R., O'Connell, J. and Sullivan, F., Molecular Dynamics on Vector Computers, J. of Computational Physics, 61 (1985).

[9] Gary, J. M., A Vectorizer for the Cyber 205 with Runtime Optimization, unpublished NIST report.

[10] Gary, J. M., On a Performance Model for Finite Difference Schemes Directed to the Alliant, unpublished NIST report.

[11] Metropolis, N., Rosenbluth, A. W., Rosenbluth, M. N., Teller, A. H., and Teller, E., Equation of State Calculations by Fast Computing Machines, J Chem. Phys. 21 (1953), pp. 1087-1092.

[12] Mink, A., Roberts, J. W., Draper, J. M., and Carpenter, R. J., Simple Multiprocessor Performance Measurement Techniques and Examples of Their Use, NBSIR 86-3416, National Bureau of Standards, (1986).

[13] Raveché, H. J., Lawrie, D. H., and Despain, A. M., A National Computing Initiative: The Agenda for Leadership, Report of the Panel on Research Issues in Large-Scale Computational Science and Engineering, SIAM (1987).

[14] Williams, G. O. and Kalos, M. H. 1984. A New Multispin Coding Algorithm for Monte Carlo Simulation of the Ising Model, J. Statistical Physics 37 (1984), pp. 283-299.

\section{Appendix- Summary of Activities}

Goals.

- Produce a taxonomy of algorithm kernels based on data motion rather than on problem type or numerical method. (Kernel process is often different from the problem statement.)

- Understand performance at machine-event level. Measure performance of kernels.

- Connect the taxonomy and measurements to real applications on real mechines. 
- Derive principles of parallel algorithm design.

Elements of the taxonomy.

- Iteration on regular grids.

- Event scheduling.

- Searching ordered structures.

- Traversing graphs. (depth-first search, spanning trees, connected components).

- Random walk.

Machine architectures.

- MIMD.

- Tightly coupled shared memory: Sequent, Alliant, Enccre.

- Loosely coupled message passing: INTEL, NCUBE

- SIMD.

- Vector: CYBER 205, CONVEX.

- Rectangular array: MPP, DAP.

- Hypercube: Connection Machine. 
Gaithersburg applications program.

(Develop taxonomy, perform measurements on kernels)

- Iteration examples.

- Ising spin simulation on CYBER 205 and MPP.

* Boolean operations used for vector compares.

* "Colors" uused for domain decomposition.

- Ising on Connection Machine.

* Use of $\log _{p}(r)$ bits for random "demons."

* Domain decomposition via 16 colors.

- Independent (chaotic) Ising model on Sequent.

* $\sin ^{-1} t$ "gambler's ruin" effect when no locking is used.

- Synchronized (colors) Ising on Sequent.

* Synchronization overhead greater than locking overhead.

- Ising on hypercube.

* Domain decomposition and chaotic boundaries (shuffling).

- Diamondsort on mesh-connected SIMD machines.

- Image algorithms.

* Hausdorff mean distance between images on DAP, MPP and Sequent.

* Shonkwiler image distance algorithm. (ADI-type method.)

* Statistical "coarsening" algorithm. (Cyclic reduction type method.)

- Searching examples.

- Dimension calculations on DAP and MPP.

* Use of bins, total samples to avoid calculation of random numbers.

* Dimension and $<\delta>$ statistic used for test of random pairs.

- Multi-processor merge for Dimension and mean Hausdorff distance calculations on Sequent.

- Diamondsort/MLG for molecular dynamics on Connection Machine.

- MIMID random numbers. 
- $O(\log n)$ merge/sort on $n^{2}$ processors.

- Balanced oct-tree search for random surface simulations.

- Event scheduling examples.

- Quicksori with heaps for fractal dimension calculations on Sequent.

- Adaptive Monte Carlo quadrature method.

- "Parking lot" problem via adaptive method with event scheduling.

- "Chaotic" Ising with waits (as in Lubachevsky).

- Graph traversal examples.

- Triangulation in 3-D by depth-first search of directed graphs.

- Shelling by depth-first search of undirected graphs.

- Random walk examples.

- Self-avoiding random surfaces simulation. (Also uses balanced tree search.) 


\section{Gaithersburg measurements program.}

(Develop non-intrusive measurement hardware and software.)

- Sequent instrumented.

- "TRAMS" software for collecting and displaying statistics developed.

* Time marking in use.

* Accumulated resource usage software and hardware completed.

- Hypercube being instrumented.

- Measurements performed on Ising iteration benchmark kernel.

- Wait times at end of sweep for synchronized Ising with "colors."

- Lock times for chaotic Ising.

- Measurements performed on quicksort with heaps.

- SIMD purchase in progress. 
Boulder applications program.

(Scale-up to real applications on large machines. Statistical studies of ${ }^{*}$ benchmark data.)

- Comparisons with University of Colorado taxonomy.

- Participation in CU/NCAR parallel computing center.

- Languages.

- FORCE. (Shared memory machines.)

- Schnabel project. (Message passing machines.)

- Iteration example.

- PDE code on Alliant and Encore.

- Scale up to large machine via MFE net.

- Searching ordered structures example.

- Neighbor table method for non-equilibrium molecular dynamics on $\mathrm{Cy}$ ber 205 .

- L. Fosdick's particle medthods.

- Contact with "Perfect" club.

- Statistical analysis of benchmark data. 
Kernels

- Iteration:

Ising. Completed, reported.

- Event scheduling.

Quicksort with heaps. Completed, reported.

- Searching.

Dimension codes completed.

Measurements of Hausdorff distance code on Sequent proceeding.

- Traversing graphs.

Codes completed.

- Random walk.

Algorithm for self-avoiding surfaces designed. Code completed.

General Results and Observations.

- Details from examples find general application. There are crossovers.

- Some architecture independence occurs in parallel iteration algorithms because of general requirement for data indepenence.

- Measurement results guide algorithm design. Communication cost dominates performance.

- Inexact SIMD "image" techniques give greatest speedup.

- Chaotic methods are important for MIMD architectures.

* Iteration via chaotic colors wins.

* Chaotic graph traversal gives triangulation and shelling.

- Event scheduling efficiency depends on number of processors doing "small" or "big" events.

Future issues

- Parallel computing vs massively parallel computing. 
- Mathematical theory.

- A co-ordinated strategy is needed for distribution and use of benchmarks. A scale-up of our kernels can give the third dimension of the "Perfect" club benchmarks. taxonomy.

- Communications benchmarks are needed. (Princeton Conference report will give some details.)

\section{Publications resulting from the project.}

- Amar, J. G., Sullivan, F. E., and Mountain, R. D., "A Monte Carlo Study of Growth in the Two-Dimensional Spin-Exchange Kinetic Ising Model", Phys. Rev. B, 37 (1988).

- Ainar, J. .G., and Sullivan, F. E., "Monte Carlo Simulation of Domain Growth in the Kinetic Ising Model on the Connection Machine," submitted to Computer Physics Communications.

- Blue, J. L., and Sullivan, F. E., "Ising Spin on a Shared Memory Machine: Computational Experience", Proc. Third SIAM Conf. on Parallel Processing for Scientific Computing, to appear.

- Beichl, I., and Sullivan, F. E., "Triangulation from the Ordertype," submitted to Discrete and Computational Geometry.

- Hunt, F. and Sullivan, F. E., "How to Estimate Capacity Dimension," proceedings of the 3rd UC Conference on Statistical Mechanics, to appear in Nuclear Physics B.

- Gary, J. M., "On a Performance Model for Finite Difference Schemes Directed to the Alliant", submitted to the J. of Parallel Computing.

- Gary, J. M., and Wang, J. "Analysis of Performance Data," submitted to SIAM J. of Scientific and Statistical Computing.

- Gary, J. M., "A Vectorizer for the Cyber 205 with Runtime Optimization," unpublished NIST report.

- Lyon, G. "Descriptive Factors for Parallel Processing Benchmarks," to appear J. Theoretical Computer Science. 
- Carpenter, R. "Performance Measurement Instrumentation..." to appear J. Theoretical Computer Science.

- Mink, A., Roberts, J. W., Draper, J. M., and Carpenter, R. J., "Simple Multiprocessor Performance Measurement Techniques and Examples of Their Use," NBSIR 86-3416, National Bureau of Standards, (1986). 

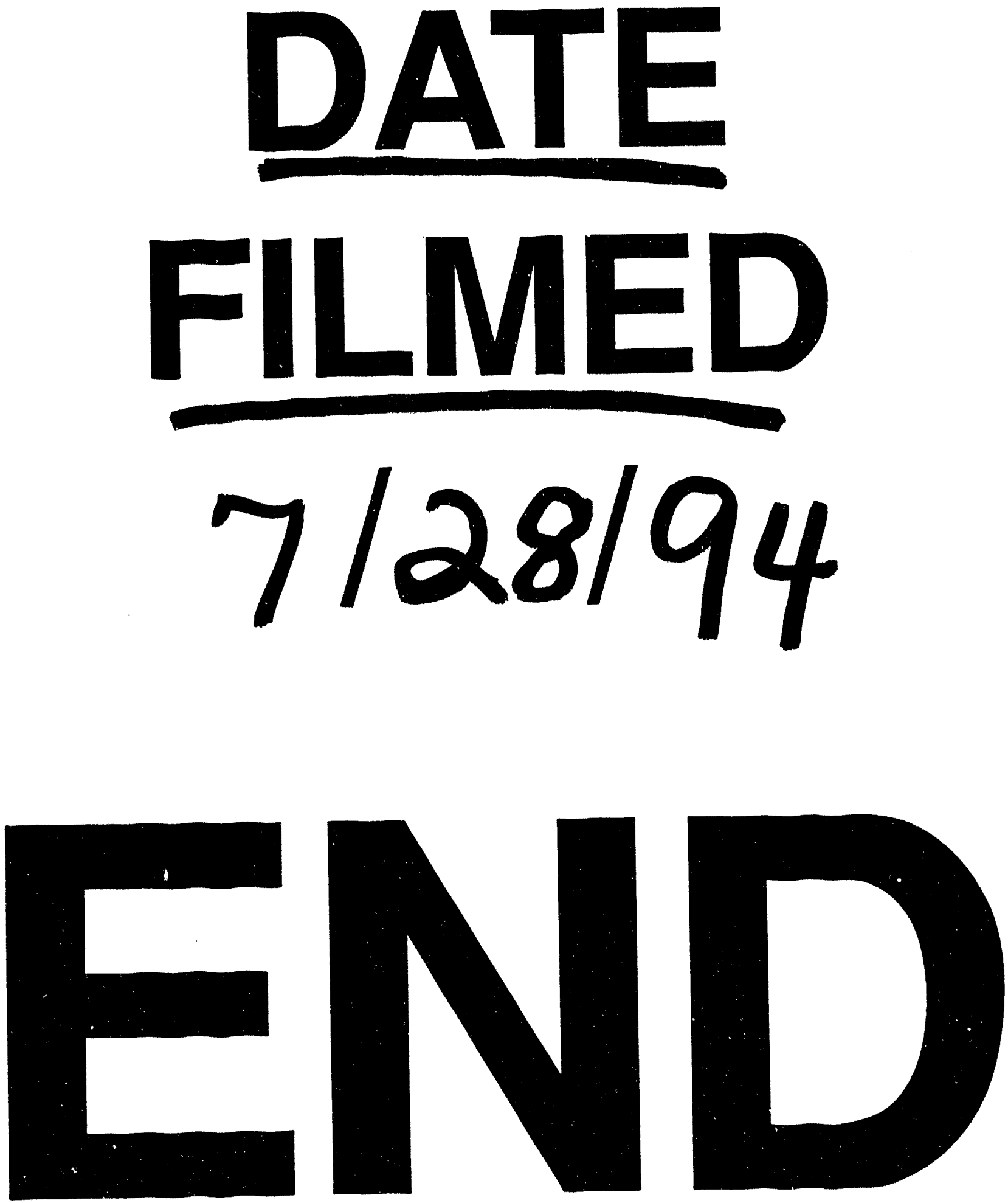
